effectiveness of the FIFA $11+S$ injury prevention program in reducing upper extremity injuries.

Results The vast majority $(97.60 \%)$ of the participants were goalkeepers. A total of $204(28.25 \%)$ of the participants were aware of the FIFA $11+\mathrm{S}$ injury prevention program, 155 $(21.46 \%)$ were implementing the FIFA $11+\mathrm{S}$ injury prevention program in their current practice. Participants who implemented the FIFA $11+S$ injury prevention program reported a positive opinion about the program efficacy, with a score of $8.19 \pm 0.93$ out of 10 .

Conclusions The awareness level was low, but the implementation rate among aware participants was acceptable. Goalkeepers and coaches reported a positive score regarding the effectiveness of the FIFA $11+S$ injury prevention program in reducing upper extremity injuries.

\section{INCREASED ACUTE EXPOSURE TO SOCCER BALL HEADING SHOWS NO RESPONSE FROM BIOCHEMICAL MARKERS FOR AXONAL INJURY}

${ }^{1}$ Kieran Austin, ${ }^{2}$ Ben Lee, ${ }^{2}$ Tessa Flood, ${ }^{6}$ Jamie Toombs, ${ }^{6}$ Mina Borisova, ${ }^{1}$ Neal Smith, ${ }^{5,6}$ Amanda Heslegrave, ${ }^{3,4,5,6}$ Henrik Zetterberg. 'Institute of Sport, University of Chichester, Chichester, UK; ${ }^{2}$ Occupational Performance Research Group, Institute of Sport, University of Chichester, Chichester, UK; ${ }^{3}$ Department of Psychiatry and Neurochemistry, University of Gothenburg, Gothenburg, Sweden; ${ }^{4}$ Clinical Neurochemistry Laboratory, Sahlgrenska University Hospital, Mölndal, Sweden; ${ }^{5}$ Department of Neurodegenerative Disease, UCL Institute of Neurology, London, UK; ${ }^{6}$ UK Dementia Research Institute, University College London, London, UK

\section{$10.1136 /$ bjsports-2021-IOC.283}

Background There is limited research quantifying the response of biochemical markers for axonal injury to an acute bout of soccer heading, especially when individual impact biomechanics are considered, and overall heading dose manipulated.

Objective To investigate if individual impact biomechanics or different heading doses affect the reaction of serum neurofilament light (NF-L).

Design Randomized control trial.

Setting Laboratory study.

Participants Forty-four male university students with no history of head injury and currently not active in head-impact sports, randomly split into a control group $(n=8), 10 \quad(n=12), 20$ $(n=12)$ or 40 -header group $(n=12)$.

Interventions (or Assessment of Risk Factors) Blood samples were taken at baseline, then six hours, 24-hours and 7-days post heading. After baseline samples, participants completed either 10,20 or 40 headers whilst impact biomechanics were quantified using a three-dimensional motion capture system sampling at $1000 \mathrm{~Hz}$. NF-L was quantified using Quanterix NF-L assay kit on the Simoa HD-1 Platform.

Main Outcome Measurements NF-L concentrations, cumulative linear and cumulative angular head acceleration. For NF-L concentrations, time, group and interaction effects were assessed via mixed-effects ANOVA. The relationship between cumulative linear and angular head accelerations and percentage change in NF-L across timepoints was assessed via Pearson product moment correlations.

Results There were no significant time, group or interaction effects for NF-L. There was no significant relationships between cumulative linear or angular head accelerations and percentage change of NF-L at any timepoint. At $6 \mathrm{hrs}, 24 \mathrm{hr}$ and 7-days post heading, control group showed $61.8 \%$, $41.7 \%$ and $30 \%$ NF-L change from baseline respectively; 10 header group $30 \%,-0.6 \%$ and $15.2 \%$ change respectively, 20 header group $-2.6 \%,-3.2 \%$ and $7.4 \%$ change respectively, and 40 header group $18.6 \%, 33.5 \%$ and $29.6 \%$ change respectively. Large inter-individual variation in NF-L response was observed.

Conclusions Individual impact biomechanics or increasing heading doses did not effect NF-L response. Further study is required to evaluate chronic loading effects of heading that also considers individual impact biomechanics.

\section{ABSTRACT WITHDRAWN}

\section{THE EFFECT OF A 90-MIN SOCCER MATCH AND FATIGUE ON ECCENTRIC HAMSTRING STRENGTH: IMPLICATIONS FOR HAMSTRING INJURY RISK}

${ }^{1}$ Georgios Kakavas, ${ }^{2}$ Afxentios Kekelekis. ${ }^{1}$ ECOSEP, Athens, Greece; ${ }^{2}$ Sport Injury clinic for prevention and rehabilitation, Crete, Greece

\subsection{6/bjsports-2021-IOC.284}

Background Hamstring injury (HI) is the most prevalent muscle injury in both amateur (van Beijsterveldt et al., 2015) and professional (Ekstrand et al., 2011; Hawkins et al., 2001) soccer (football) players. Worryingly, the annual incidence of $\mathrm{HI}$ increased by 2.4\% annually between 2010 and 2014 (Ekstrand et al., 2016)

Setting Professional football.

Patients (or Participants) Twenty-two semi-professional football players (Mean \pm SD: Age: $19.3 \pm 2.9$ year; Height $185.0 \pm$ $8.7 \mathrm{~cm}$; Body Mass $81.6 \pm 6.7 \mathrm{~kg}$ ) participated in this study. Design All players were right dominant (defined as their preferred 'kicking' leg). Subjects were included in the study if they were not injured or rehabilitating from an injury at the time of testing.

Main Outcome Measurements The eccentric hamstring strength of players was examined using the Nordbord (Vald Performance, Queensland, Australia) device prior to soccer match and immediately following 90 minutes of football friendly match. The season period of the research project was the second half of the season.

Results There was a significant difference of moderate effect in eccentric strength before a match $(\mathrm{M}=306.91, \mathrm{SD}=$ 59.26) compared and to after the match (mean $=277.77 \pm$ $\mathrm{SD}=60.35 \mathrm{p}=.000$, Cohen's $\mathrm{d}=.50$ ) (table 1). The eccentric force before training (mean $=306.91 \pm \mathrm{SD}=$ 59.26) and after training $(\mathrm{M}=277.77, \mathrm{SD}=60.35$ demonstrated a significant moderate effect $(\mathrm{p}=.000$, Cohen's $\mathrm{d}=$ $.50)$.

Conclusions Etiological risk factors attributed to an increased risk of muscular injury include poor muscular strength, particularly eccentric strength deficits, and ipsilateral muscular strength imbalances. Resistance to fatigue and eccentric strength, particularly at high speeds should be given greater consideration in conditioning for soccer. The small number of participants limits the global generalization of our findings. However, this limitation is tempered by the inclusion of professional players in our study. 